\title{
CREATING DIGITAL ACTIVITY SCHEDULES TO PROMOTE INDEPENDENCE
}

\section{Creating Digital Activity Schedules to Promote Independence and Engagement}

Kassidy S. Reinert, Thomas S. Higbee, and Lyndsay D. Nix

Utah State University

Author Note

Correspondence concerning this article should be addressed to Thomas S. Higbee, Department of Special Education and Rehabilitation, Utah State University, Logan, UT 84322-2865 USA.

Email: tom.higbee@usu.edu 


\begin{abstract}
Photographic activity schedules have been demonstrated to be effective in helping individuals with autism and other developmental disabilities to learn how to complete both simple and complex sequences of activities without prompting from adults. While the majority of research studies demonstrating the effectiveness of activity schedules have used schedule books composed of static printed pictures attached to physical pages, recently, researchers have begun to demonstrate the effectiveness of technology-based activity schedules. In the current paper, we provide a task analysis for creating both simple and complex digital activity schedules using Google Slides, a freely available, web-based technology that operates on a variety of digital platforms. We also provide suggestions for how behavior analysts can train parents to use this technology with their children using telehealth procedures.
\end{abstract}

Keywords: photographic activity schedules, technology-based learning, parent training 


\section{Creating Digital Activity Schedules to \\ Promote Independence and Engagement}

\section{Editor's Note}

This manuscript is being published on a highly expedited basis, as part of a series of emergency publications designed to help practitioners of applied behavior analysis take immediate action to adjust to and mitigate the COVID-19 crisis. This article was submitted on 4/3/20 and received final acceptance on $04 / 6 / 20$. The journal would like to especially thank Dr. Thomas Szabo for his expeditious review of the manuscript. The views and strategies suggested by the articles in this series do not represent the positions of the Association for Behavior Analysis, International or Springer Nature.

\section{Introduction}

A photographic activity schedule is a set of pictures or words that prompts an individual to engage in a sequence of specific behaviors (McClannahan \& Krantz, 1999). Researchers have shown activity schedules to be an effective way to teach individuals with disabilities a variety of skills including independent completion of work tasks (Carson et al., 2008), independent solitary play for children with autism (MacDuff et al., 1993), and both simple and complex social play for children with autism (Akers et al., 2018; Betz et al,. 2008; Brodhead et al., 2014). These studies demonstrate that activity schedules promote independence, increase learner engagement with appropriate activities, and decrease reliance on prompts. Therefore, these tools are ideally suited for situations, like the current COVID-19 crisis, where access to direct, professional-led learning may be reduced and clients may be provided with longer periods of "down time" than under typical circumstances.

Activity schedules are an attractive tool for promoting independence not only because of 
their effectiveness but also because of the broad range of individuals with whom they can be used and wide array of skills they can be used to promote. They require relatively few prerequisite skills and conditions in order to be effective (e.g., picture-object correspondence, acceptance of physical prompts, identified reinforcers) and can be used to teach sequences of behavior as simple as independent play sequences (MacDuff et al., 1993) and as complex as group social games such as hide-and-seek (Akers et al., 2018). Importantly, researchers have demonstrated that parents can be taught, via technology, to implement activity schedule teaching procedures with high degrees of fidelity (Gerencser et al., 2017).

To date, most activity schedules used in published studies have been composed of threering binders with pictures attached to individual pages or strips of pictures on a single page. While inexpensive, binder or paper-based schedules may have some disadvantages, such as being cumbersome to transport (Carlile et al., 2013) and, perhaps more importantly, potentially socially stigmatizing, as same-aged peers would likely not be carrying around books during activities (Giles \& Markham, 2017).

Given technology advances that have made portable electronic devices, such as tablet computers, more readily available to teachers and families of individuals with disabilities, there may be advantages to using digital schedules that could be displayed on these devices. First, scanned or photographed images of activities could be easily moved around within activity schedules, reducing the cost and effort of printing and laminating images for inclusion in binderbased or single-page printed schedules. Second, digital schedules could easily be shared across multiple devices. For example, copies of the schedule could be updated by a teacher or therapist and then emailed to a parent for use at home. Finally, tablet computers are now ubiquitous in school, work, and home settings, thus reducing potential stigmatization for digital activity 
schedule users.

Multiple researchers have investigated the effectiveness of various forms of digital activity schedules delivered on electronic devices including: computers running Microsoft PowerPoint (Rehfeldt et al., 2004); iPods, to increase on task behavior (Carlile et al., 2013); video clips to increase dramatic play (Dauphin et al., 2004); iPads to increase time on task and decrease transition time during centers (Gourwitz, 2014); and iPads to promote completion of leisure activities (Giles \& Markham, 2017). One published study has also indicated that, for some participants, digital activity schedules may be preferred over binder-based schedules (Giles \& Markham, 2017). While further research on the use of digital activity schedules is certainly warranted, a sufficient body of research exists to demonstrate the potential benefits of this technology. Given the portability of digital activity schedules and the potential for behavior analysts to be able to design digital activity schedules and transmit them electronically to parents or caregivers for use with their children at home, we believe that they are ideally suited to the current therapeutic environment caused by the COVID-19 pandemic that limits direct contact between behavior analysts and the clients and families with whom they work. Thus, the purpose of this paper is to provide a detailed task analysis that both behavior analysts and parents can use to create and modify digital activity schedules using Google Slides, a freely available web-based program that works on a variety of digital devices and platforms.

\section{Brief Activity Schedule Primer}

While detailed descriptions of how to create and build independence using activity schedules are available elsewhere (e.g., Higbee \& Brodhead, 2016, Higbee \& Sellers, 2017; McClannahan \& Krantz, 1999), a brief summary of how to select activities for inclusion in a digital activity schedule and standard prompting/teaching techniques is included here. We refer 
the reader to the cited papers for more detailed descriptions and instructions.

\section{Selecting Activities and Arranging the Environment}

Specific activities to be included in a beginning activity schedule, including a digital one, should be moderately preferred, close-ended (have a clear beginning and end), relatively brief, and already independently performed by the learner, as the focus during initial activity schedule training is on how to follow the schedule rather than on the activities themselves (Higbee \& Sellers, 2017). Beginning activity schedules are usually composed of at least 2-3 activity pages plus a terminal reinforcer page (typically a small amount of a preferred edible) at the end. Additional activities can be added as the learner demonstrates mastery of the schedule to form progressively longer response chains. The sequence of activities should be changed frequently to ensure that the learner's behavior comes under the control of the pictures displayed as opposed to becoming a sequence of rote behaviors under proprioceptor control of sensations repeated in a performance chain.

Activity schedules can be used in multiple environments. However, beginning play/leisure activity schedules often take place at a table or desk that is large enough to both hold the schedule and provide enough room to complete the activities (the floor could also be used in the absence of a desk or table). Activity materials are typically placed on a shelf near the desk/table. They could also be placed directly on the table if it is large enough to hold all materials and still have a clear workspace to complete activities. Alternatively, materials could be placed on the floor near the table. When learners are first beginning to use activity schedules, the idea is to have the materials close at hand to reduce travel time and the potential for distraction. Once learners have mastered the basic components of activity schedule following, materials can be placed in their natural locations. 


\section{Sequence of Activity Schedule Following}

Learners are taught, through a procedure called "graduate guidance," described in the next section on "Prompting Procedures," to follow the activity schedule. The following sequence of behaviors constitutes "following the schedule:"

1) retrieve the schedule (obtain the tablet or other digital device) and place it on the table/floor where activities will be completed

2) open the schedule file/program (steps 1 and 2 may be completed by a parent/teacher for a digital schedule, depending on the technology level of the learner)

3) touch the picture of the activity (an observing response)

4) obtain the materials necessary for completing the activity (e.g., retrieve the pictured puzzle from a shelf)

5) complete the activity

6) return the materials back to their original location

7) "turn the page" on the digital schedule to display the next activity by touching/clicking on the arrow in the bottom right corner of the screen

8) repeating steps 3-7 until reaching the terminal reinforcer page and consuming the reinforcer

9) returning the digital device to its original location/closing the program.

\section{Prompting Procedures and Error Correction}

In an effort to reduce dependence on adult-provided verbal prompts and instructions, learners are taught to follow activity schedules exclusively via physical prompts delivered from behind the learner, so as not to block the learner's view of the relevant visual stimuli that should 
control their behavior (McClannahan \& Krantz, 1999). The only verbal instruction given to the learner should be the initial instruction to begin completing their schedule (e.g., "It's time to do your activity schedule. Here you go!"'). After that, the person assisting the learner to follow the schedule should not provide any additional verbal instructions, verbal prompts, or conversation until the schedule is completed (unless one of the included activities is a social activity during which verbal interaction would be appropriate). The amount of physical prompting provided will depend on each individual learner's abilities but will often begin with hand-over-hand guidance that should be provided in a way that allows the learner to complete the schedule with as few errors as possible. This physical guidance should be faded as quickly as possible to avoid prompt dependence. A typical fading sequence would begin with hand-over-hand guidance that is progressively faded to physical prompts at the wrist, forearm, elbow, and shoulder, followed by gradually increased physical distance between the prompter and learner. We have found that light physical prompts from behind at the shoulders are particularly effective for guiding learners back and forth between the schedule and activity materials. Fading can take place dynamically within sessions or can be scripted across sessions. One practical method for prompt fading that has been particularly useful when parents are the primary implementers is that used by Gerencser et al., (2017), where test sessions are periodically run using a $5 \mathrm{~s}$ prompt delay procedure to determine the steps a learner can complete independently. Prompts the learner needed to completed the task are recorded on a data sheet, which then serves as a teaching guide for sessions until the next test session is conducted (see Appendix 2 for a sample data sheet). Behavior analysts who have the capacity to view sessions live through video conference technology could also provide parents with fading suggestions in real time.

When learners make errors, the type of error will determine how the 
implementer/prompter should respond. If the learner begins to drift off task or begins to engage in stereotypy with schedule materials, the implementer/prompter should simply interrupt this behavior and physically redirect the learner to complete the activity correctly. However, if the learner obtains the incorrect materials after touching the picture representing those materials, they should be redirected back to the schedule, physically prompted to touch the picture of the materials again, and physically guided to obtain the correct materials. This helps ensure that learner behavior comes under the control of the schedule.

\section{Advanced Activity Schedule Components}

Once learners have mastered following basic activity schedules with brief, close-ended activities, more complex activities can be introduced. For example, implementers can add choice pages in which the learner can select from two or more available activities. On a close-ended activity schedule that a learner previously mastered, choice pages could be added to the schedule to replace one or more of the close-ended activities. Learners can also be taught to set and respond to digital timers using visual cues in order to include open-ended activities in the digital schedule. Red colored circles representing the number of minutes for the activity as well as a green circle representing the start button are placed below the picture of the open-ended activity. The minute button on the digital timer is also colored red and the start/stop button colored green. The learner is physically prompted to touch each circle below the picture and the corresponding colored button on the timer to set and start the timer. Responding to these more complex activity schedule pages can often be taught via graduated guidance within activity schedule sessions. If a learner requires more practice, modeling and teaching sessions with timers and choice pages could occur outside of activity schedule sessions (Higbee \& Sellers, 2017; McClannahan \& Krantz, 1999). 


\section{Data Collection}

Learner performance can be measured by scoring steps from the above sequence that were performed independently by the learner. A sample data sheet is included in Appendix 1. Whereas collecting data during each teaching session would be ideal during initial stages of learning, Gerencser et al. (2017) suggests test sessions that may provide sufficient data to make decisions about prompt levels. An example of the data sheet that is similar to the one used by Gerencser et al. is provided in Appendix 2. Also, when parents are implementing activity schedules, behavior analysts could score performance viewed live through videoconferencing technology or via recorded sessions sent electronically by parents.

\section{Digital Activity Schedule Template Task Analysis}

The following task analysis uses the web-based program, Google Slides, to create digital activity schedules. Google Slides is a freeware program that works on a variety of digital devices and operating systems. More information about Google Slides can be found at:

https://www.google.com/slides/about/. A flowchart of essential steps in the task analysis is provided in Appendix 3 to guide the reader and assist with troubleshooting.

\section{Creating a Google Slides Presentation}

1. Open a web browser and sign into Google account.

2. Navigate to https://docs.google.com/presentation.

3. Create a new blank presentation by clicking the multicolored plus sign in the navigation bar near the top of the page.

4. Click "Untitled presentation" in the top left corner and type Activity Schedule Template. 


\section{Activity Schedule Set-up \& Cover (Fig. 1)}

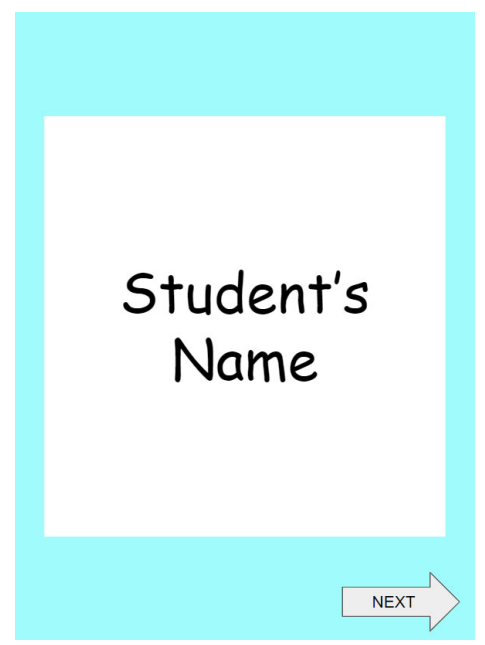

Fig. 1 Activity Schedule cover

1. Click "File" and navigate to "Page setup" near the bottom of the list.

2. Resize page by clicking "Widescreen 16:9." Select "Custom" and change dimensions to 5.5 X 8.5 inches. Press "Apply."

3. Click "Background" on the toolbar and select a desired color for the schedule background. Press "Add to theme" and then select "Done."

4. Select the subtitle text box and press the "Delete" key.

5. Click on title text box. Blue squares should appear on the text box as shown in Fig. 2. Resize title text box by dragging down the center bottom square to make a large rectangle centered on the page.

a. Google Slides provides red guidelines (Fig. 2) that aid with centering shapes, objects, etc. These guidelines pop up when an item is centered horizontally and/or vertically within a slide or with adjacent item(s).

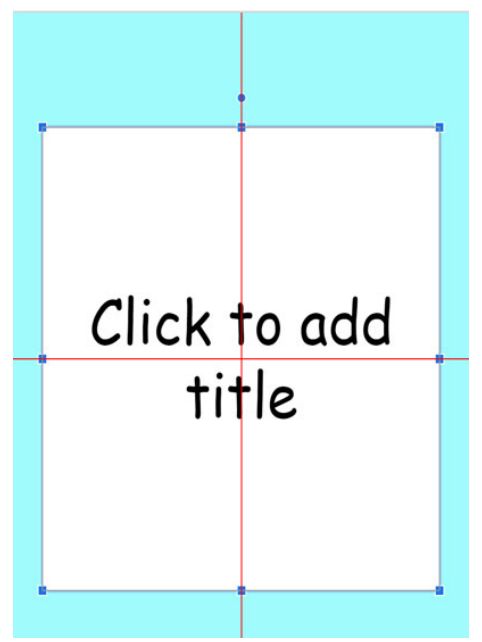

Fig. 2 Google Slides editing tools: blue squares to adjust size and red guidelines to center 
6. Select title text box and change the background to white by clicking the "Fill color" icon (Fig. 3).

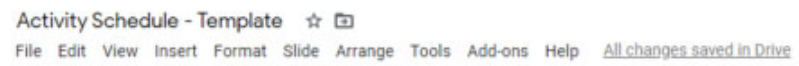

Fig. 3 Fill color icon

7. Select "Click to add title" and type "Student's Name."

8. While title text box is selected, click on the "Align" icon (Fig. 4). Center text horizontally by clicking the "Center" icon and vertically by clicking the "Middle" icon.

Activity Schedule - Template में

File Edit View Insert Format Slide Arrange Tools Add-ons Help All changes saved in Drine

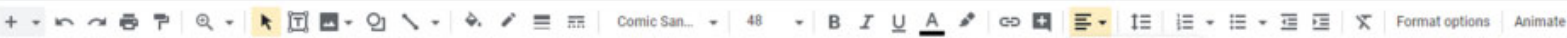

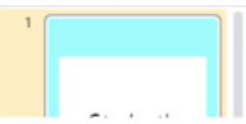

$\overline{\bar{\nabla}} \equiv \equiv$

$\overline{\mathrm{f}} \stackrel{\perp}{\mathrm{L}}$

Fig. 4 Formatting Tools: Align, Center, and Middle

9. Select "Shape" tool and select "Right Arrow" as displayed in Fig. 5. Draw in the bottom right corner of the page.

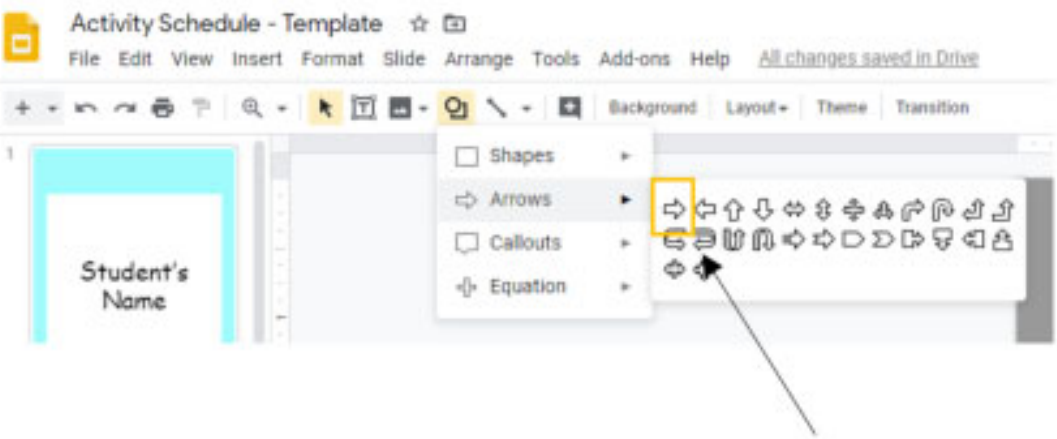

Fig. 5 Selecting a "Right Arrow"

10. Double click on the arrow and type NEXT in the center of the arrow. The activity schedule cover should look like Fig. 1. 


\section{Creating Close-Ended Activity Pages (Fig. 6) \& Terminal Reinforcer Page (Fig. 7)}

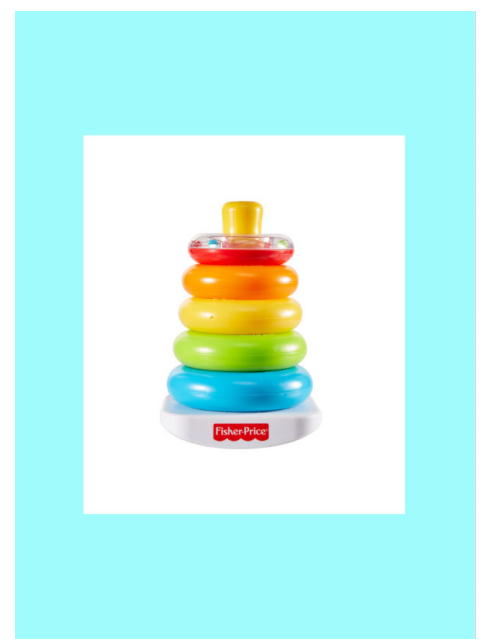

Fig. 6 Close-ended activity page

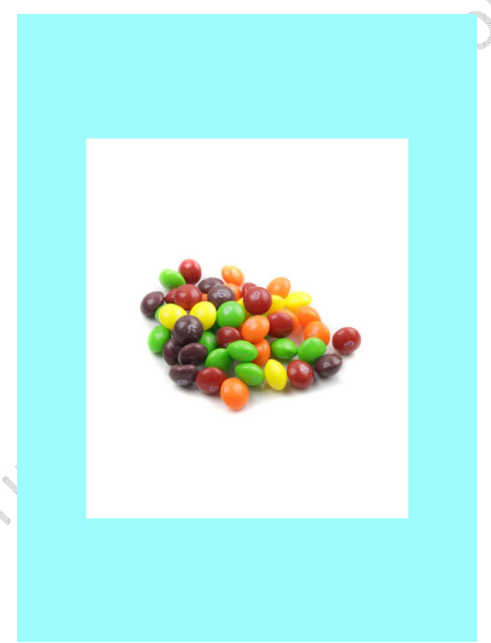

Fig. 7 Terminal reinforcer page

1. Create a new blank page by clicking on the last page in the left column and pressing the "Enter" key.

2. Delete all text boxes.

3. Insert a large white square in the center of the page.

4. Insert picture of close-ended activity using the "Insert Image" icon (see Fig. 8) to import desired pictures of activity schedule toys.

Fig. 8 Insert image icon 
5. Center and resize picture to fit in the white square area.

6. Repeat steps 1-5 to create multiple close-ended activity pages and one terminal reinforcer page. The close-ended pages of the activity schedule should look like Fig. 6 and the terminal reinforcer page should look like Fig. 7.

7. After creating all pages in a schedule, move the terminal reinforcer page to the last page in the schedule.

\section{Creating Simple Navigation between Slides}

1. Navigate to cover page.

2. Select "Shape" tool (Fig. 9) and draw a large rectangle over the entire page.

A. Activity Schedule - Template के $\square$

File Edit View Insert Format Slide Arrange Tools Add-ons Help Allchangessaved in Drive

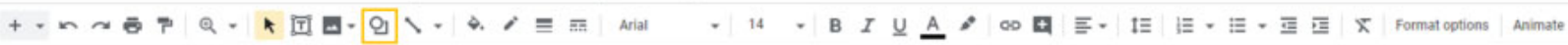

Fig. 9 Shape tool icon

3. Right click on the rectangle. Navigate to "Order" then "Send Backward." Now only the next arrow should be visible on the page.

4. Select the "Next arrow" and click the "Insert Link" icon (Fig. 10) in the toolbar. A white pop-up bar will appear on the screen. Click on "Slides in this presentation." Select "Next Slide" and press "Apply." This will now make it so clicking or pressing the next arrow during a presentation transitions to the next slide.

a. Please note that the pop-up dropdown menu may run below the bottom of the browser page and may not fully be visible. In order to view all link options, resize object or move to a higher location on the page. Perform link operation and then return to previous size and location.

Activity Schedule - Template में

File Edit View Insert Format Slide Arrange Tools Add-ons Help Allchangessaved in Drive

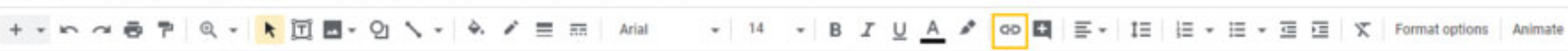

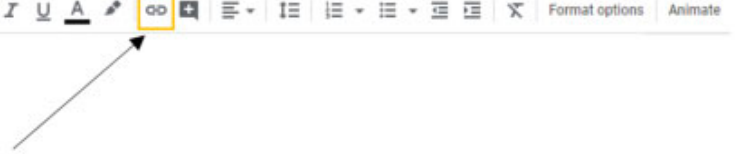

Fig. 10 Insert link icon

5. Select the rectangle and navigate to the "Insert Link" icon (Fig. 10) in the toolbar. A white pop-up bar will appear on the screen. Click on "Slides in this presentation," select current slide number, and press "Apply." This will disable all clicks or presses anywhere on the page other than a click on the arrow.

6. Click on the large rectangle and change "Color fill" and "Border color" (Fig. 11) to transparent. 
Activity Schedule - Template के

File Edit View Insert Format Slide Arrange Tools Add-ons Help Allchangessaved in Drive

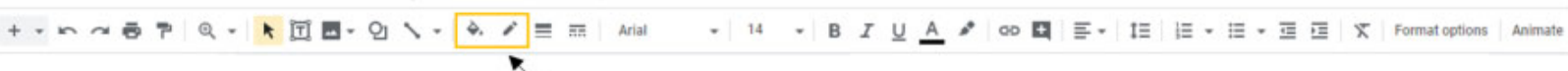

Fig. 11 Fill color icon and border color icon

7. "Copy" the large rectangle from the cover page and "Paste" on additional close-ended and terminal reinforcer pages.

8. Then copy "Next arrow" from the cover page and paste to additional close-ended and terminal reinforcer pages.

9. Completed close-ended slides can also be replicated using "Copy" and "Paste." Links will continue to work.

a. To edit the copied slides, select the large transparent rectangle and move out of the way in order to edit/replace pictures. After editing, return the large transparent rectangle to original position.

10. Go to "Present" icon (Fig. 12), located on top right corner of tool bar, to view and navigate slides.

a. Navigate through the presentation by clicking on the "Next arrows." They should advance to the next slide in the sequence.

i. If an arrow does not advance correctly, check the links by clicking on the arrow. When clicked on, each arrow should display a white link bar below with the words Next slide. If the slide linked to the arrow is incorrect, click "Remove link" and return to step 4 in Creating Simple Navigation between Slides.

ii. If there is no link, return to step 4 in Creating Simple Navigation between Slides.

b. After all arrow transitions are working correctly, restart the slideshow. Check that no other transitions occur when clicking elsewhere on the screen (anywhere other than the arrows).

i. If transitions occur, return to step 5 in Creating Simple Navigation between Slides. 


\section{Creating Choice Pages (Fig. 13)}

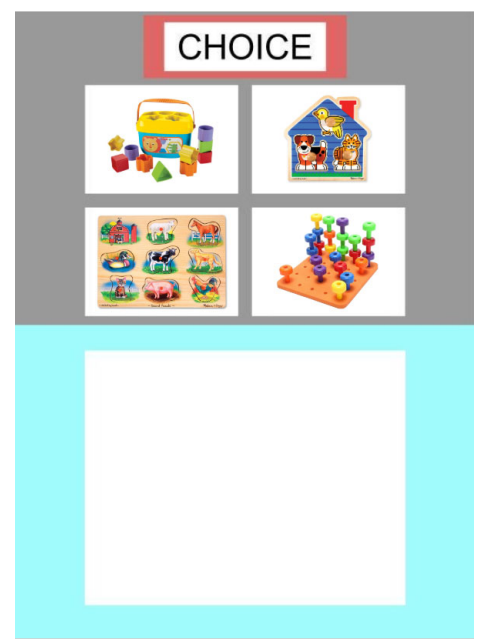

Fig. 13 Choice page with four toy options

1. Create a new blank page by clicking on the previous page in the left column and pressing the "Enter" key.

2. Delete all text boxes.

3. Select "Shape" tool icon (Fig. 9) and draw a rectangle that completely covers the top half of the page.

4. Click on the rectangle and change the color using the "Fill color" icon (Fig. 3) to a light grey.

5. Select "Shape" tool (Fig. 9) and draw a white rectangle in the lower half of the page, leaving a $1 / 2$ inch colored margin around the edge.

6. Draw one white small rectangle in the top half of the page.

7. "Copy" the small rectangle and "Paste" it three times.

8. Arrange the four small rectangles in a $2 \times 2$ grid as show in Fig. 13 using the red guidelines (Fig. 2).

9. Create a red rectangle at the top of the page using the "Shape" tool (Fig. 9). Use the red guidelines (Fig. 2) to center on the page.

10. Select the "Text box" icon (Fig. 14).

Activity Schedule - Template के

File Edit View Insert Format Slide Arrange Tools Add-ons Help Allchanges savedin Drivg

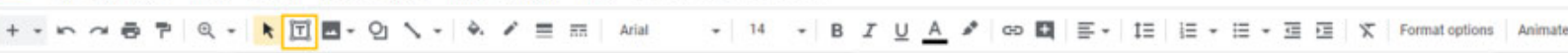

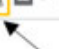

Fig. 14 Text box icon

11. Resize the text box to fit within the red rectangle (leaving a red border around it) and center using the red guidelines (Fig. 2).

12. Click on the text box and type CHOICE. 
13. Center text horizontally and vertically using the "Align" icon (Fig. 4).

14. Click on the text box, click the "Color fill" icon (Fig. 3), and select white to fill the text box with a white background.

15. Select the "Choice page" in left navigation bar.

16. In the "File" menu, navigate to the "Download" section, and select "PNG Image (.png, current slide)" (Fig. 15).

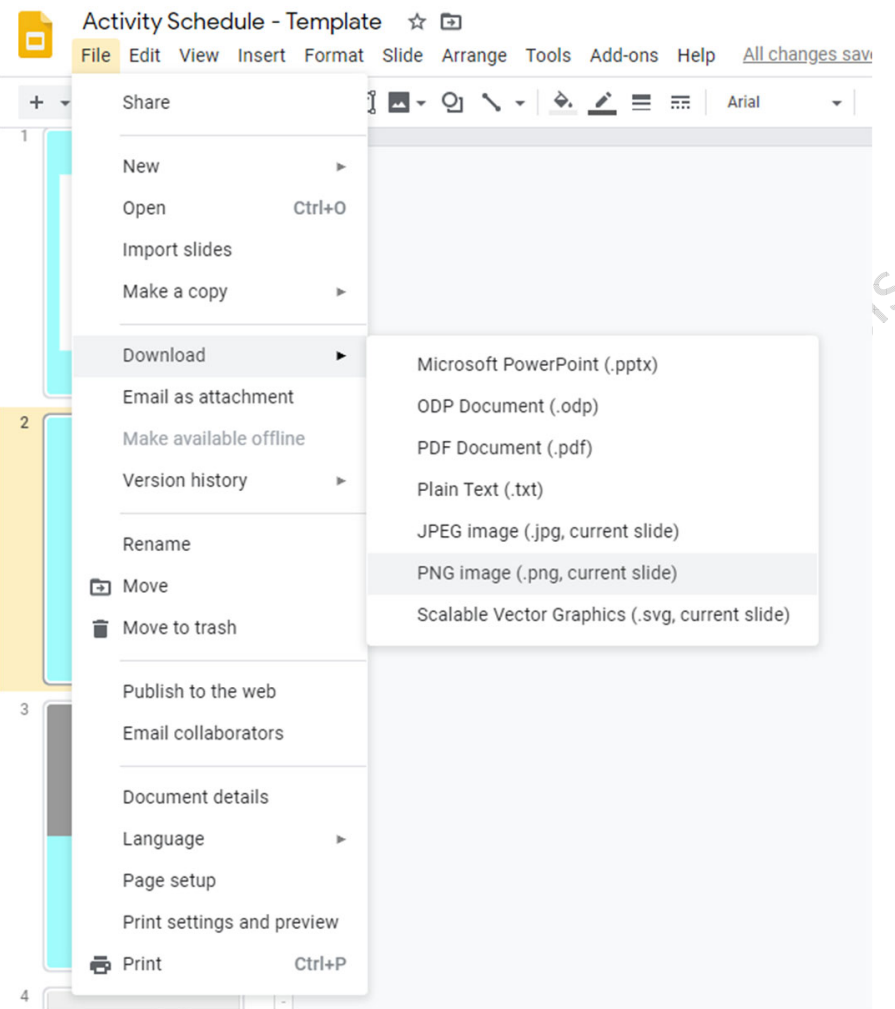

Fig. 15 Downloading a PNG file

17. A Save page may appear, or it may automatically download.

a. If given the option to save, title the .png file as Activity Schedule Template Choice Page, select desired location, and press "Save."

18. Select Choice page in left navigation bar, right click, and select "Delete."

19. Create a new blank page by clicking on the previous page in the left column and pressing the "Enter" key.

20. Select new page and click on "Background" button on the top navigation bar (Fig. 16).

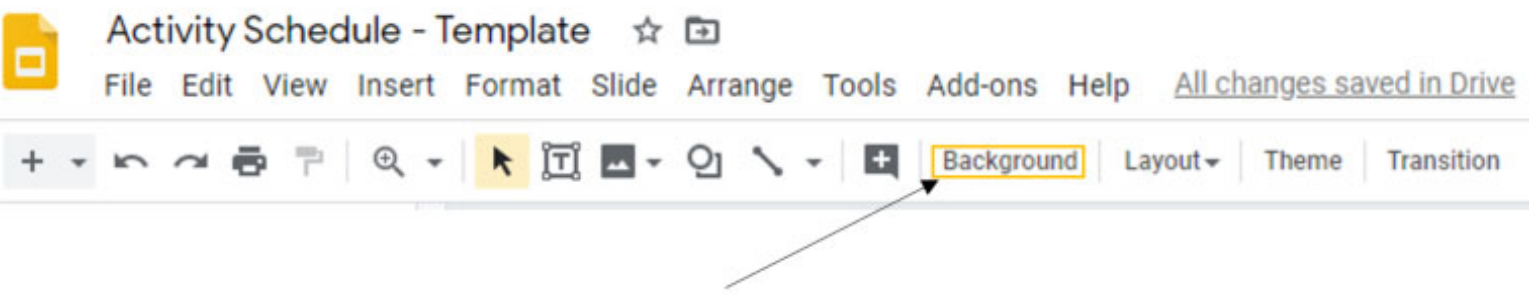

Fig. 16 Background button 
21. Select "Choose image."

22. Click the "Browse" button and select the "Activity Schedule Template Choice Page.png" or drag the .png file into specified area.

23. Press "Done." This now makes a template for a choice page that cannot be edited or moved.

24. Delete the blank text boxes on the page.

25. Insert four toy pictures using "Insert image" icon (Fig. 8). Resize pictures and place into the four white rectangles in the top half of the page. After placing pictures, the choice page should look like Fig. 13.

26. Right click "Choice page" in the left navigation bar and press "Copy."

27. Right click in the left navigation bar and press "Paste" four times. Now there should be five identical choice slides (Fig. 17). 


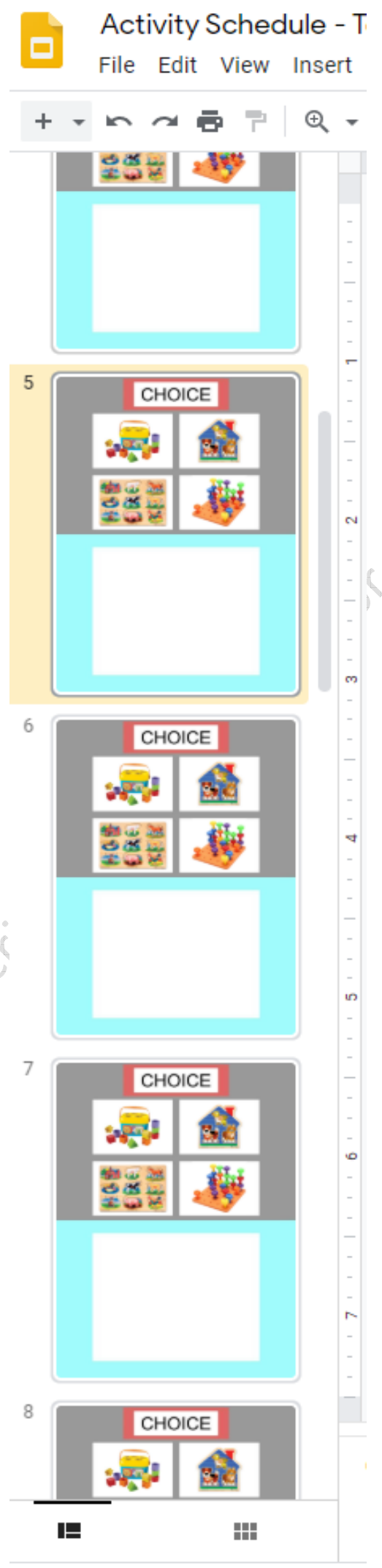

Fig. 17 Five identical slides in left navigation bar 
28. Select the second choice page in the left navigation bar.

29. Right click on one of the four pictures in the top four rectangles and select "Copy."

30. Right click on the page and press "Paste."

31. Select the copied picture. Move it to the large white rectangle in the bottom half of the page and resize the picture using the blue squares (Fig. 2). The page should now look like the page displayed in Fig. 18.

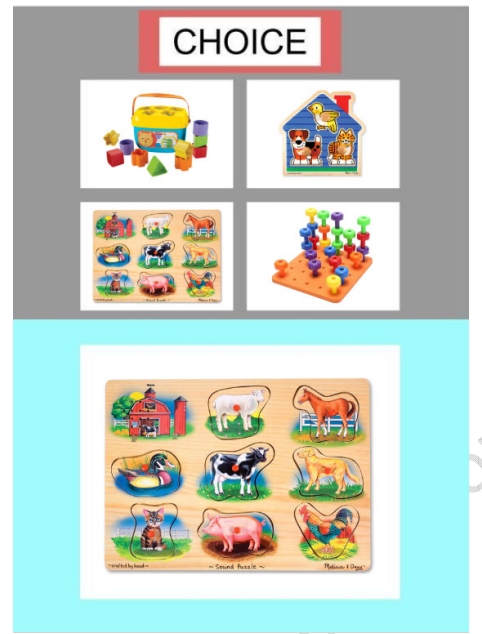

Fig. 18 Choice page with four options and puzzle selected

32. Repeat steps $28-31$ for remaining three toys and pages.

\section{Creating Navigation for Choice Pages}

1. In the left navigation bar, navigate to the last choice page with a large picture in the lower rectangle (Fig. 18) and press the "Enter" key to create a blank page. Title this new slide "Blank."

2. Navigate to the first choice slide with an empty lower rectangle (Fig. 13). There should not be a "Next" arrow on this page.

3. Draw a large rectangle using the "Shape" tool (Fig. 9) over the entire page.

4. Right click on the rectangle. Navigate to "Order" and then select "Send to Back." Now only the four toy pictures should be visible on the page.

5. Click on the large rectangle and navigate to the "Insert Link" icon (Fig. 10) in the toolbar. A white pop-up bar will appear on the screen. Click on "Slides in this presentation" and select the current slide number. Press "Apply."

6. Click on the large rectangle and change "Color fill" and "Border color" (Fig. 11) to transparent.

7. Right click on rectangle and select "Copy."

8. Navigate to remaining choice pages and "Paste" the transparent rectangle on all four pages. 
a. To ensure correct navigation, click on the transparent rectangle on each subsequent choice page. The rectangle on each slide should display a white link bar on the lower left corner with the current slide number. If the slide linked to the rectangle is incorrect, click "Remove link." Return to step 5 in Creating Navigation for Choice Pages.

9. Navigate to the first choice slide with an empty lower rectangle (Fig. 13).

10. Click on one toy picture and click on the "Insert Link" icon (Fig. 10) in the toolbar. A white pop-up bar will appear on the screen. Click on "Slides in this presentation" and select the corresponding slide number that has the identical toy picture enlarged in the lower half of the page. Press "Apply."

11. Repeat step 10 for the remaining three toy pictures.

a. To ensure correct navigation, click on each picture in the first choice slide. When clicked on, each picture should display a white link bar below with the corresponding slide number (see flow chart in Appendix 3). If the slide linked to the small picture is incorrect, click "Remove link." Return to step 10 in Creating Navigation for Choice Pages.

12. Navigate to the Student's Name page (cover of the presentation).

13. Right click on the "Next arrow." Press "Copy."

14. In the left navigation bar, select the second choice page (the first slide with a large toy picture in the lower rectangle) (Fig. 18), and press "Paste."

15. Select the "Next arrow" and click the "Insert Link" icon (Fig. 10) in the toolbar. A white pop-up bar will appear on the screen. Remove the previous link by clicking on the "X." Click on "Slides in this presentation." Select the "Blank" slide and press "Apply." This will now make it so clicking or pressing the next arrow during a presentation transitions to the next slide.

16. Right click on the "Next arrow." Press "Copy."

17. "Paste" a copy of the arrow on the remaining three choice pages. Each of these pasted arrow's navigation should automatically be linked to transition to the blank slide.

a. To ensure correct navigation, click on the "Next arrow" on each subsequent choice page. When clicked on, each next arrow should display a white link bar below with the blank slide number. If the slide linked to the arrow is incorrect, click "Remove link." Return to step 15 in Creating Navigation for Choice Pages.

18. Go to "Present" icon (Fig. 12), located on top right corner of tool bar, to view and navigate choice slides.

a. If there are errors with transitions from the initial choice slide to subsequent choice pages, refer to step 11 in Creating Navigation for Choice Pages.

b. If there are errors with the transition arrows on the subsequent choice pages, refer to step 17 in Creating Navigation for Choice Pages.

c. After all arrow and picture transitions are working correctly, restart the slideshow. Check that no other transitions occur when clicking elsewhere on the screen (anywhere other than the arrows and the first choice page pictures). If transitions occur, return to step 8 in Creating Navigation for Choice Pages. 


\section{Three-Minute Timed Activity Pages (Fig. 19)}

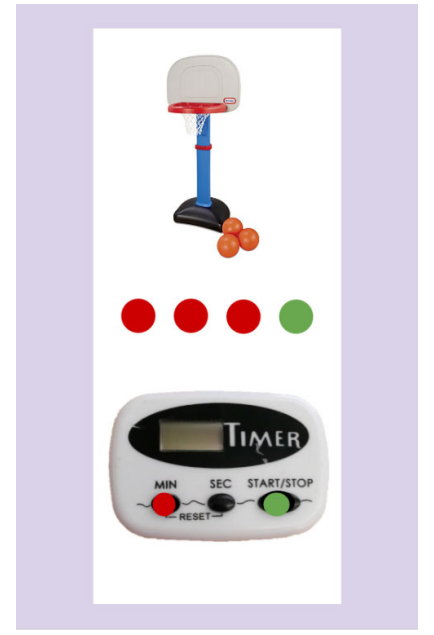

Fig. 19 Timer page

1. Select the "Blank" slide. This is the final slide of the choice page sequence.

2. Delete all text boxes on page.

3. Select "Shape" tool (Fig. 9). Draw a long white rectangle and center it on the page (Fig. 20) using the red guidelines (Fig. 2).

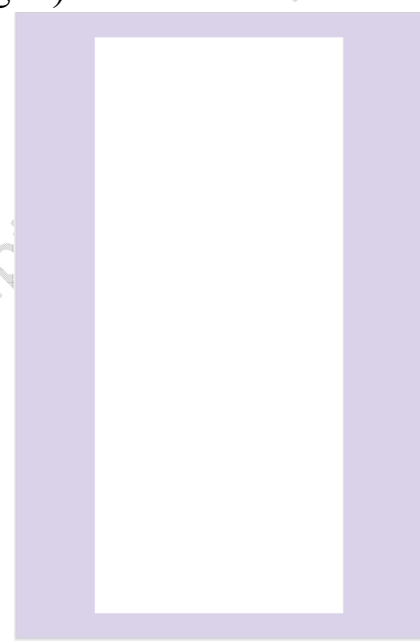

Fig. 20 Long rectangle in center of page

4. Import a picture (Fig. 5) of a timer that has both minute and start buttons (see example image in Appendix 4). Place the timer on the lower half of the page inside the white rectangle (Fig. 21). 


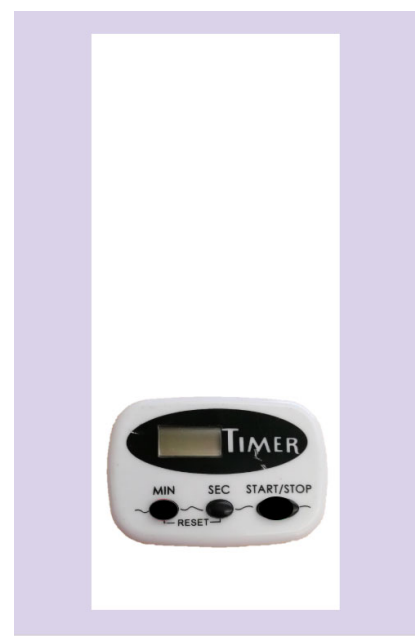

Fig. 21 Timer on page

5. Select "Shape" tool (Fig. 9) and draw one red circle. Pressing shift while drawing the circle will keep it perfectly round. Right click on the circle, select "Copy," and "Paste" it three times.

6. Space all four circles evenly in the middle of the page (Fig. 22) using the red guidelines (Fig. 2).

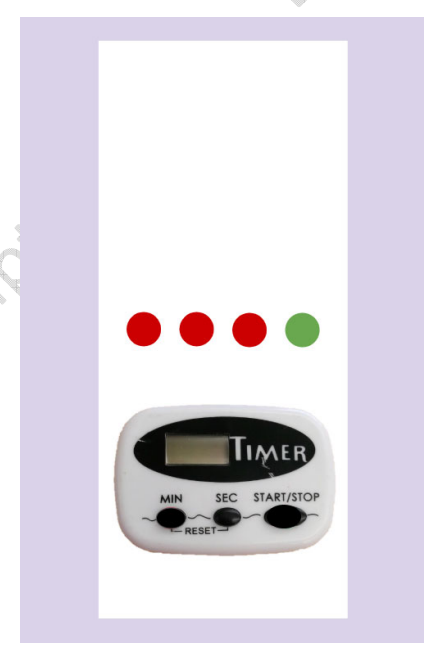

Fig. 22 Circles on page

7. Select the fourth red circle. Select the "Color fill" icon (Fig. 3) and change the color to green.

8. Select the timer slide in the left navigation bar.

9. In the "File" menu, navigate to the "Download" section, and select "PNG image (.png, current slide)" (Fig. 15).

10. A Save page may appear or it may automatically download.

a. If given the option to save, title the .png file as Activity Schedule Template Three Minute Timer, select desired location, and press "Save." 
11. Please note: Create timer page templates with longer or shorter activity durations (e.g., two minutes, four minutes) by increasing or decreasing the number of red circles on the page. Copy the current slide to a new Google Slides document and save as Activity Schedule Timer Page Template. This will allow the ability to edit and create other .png file timer pages.

a. Click "File" and navigate to "Page setup" near the bottom of the list.

b. Resize page by clicking "Widescreen 16:9." Select "Custom" and change dimensions to 5.5 X 8.5 inches. Press "Apply."

c. Navigate to "Activity Schedule Template" slideshow.

12. Select timer page in left navigation bar. Select and delete all individual items on the page.

13. Select the blank page and click on "Background" button on the top navigation bar (Fig. 16).

14. Select "Choose image."

15. Click the "Browse" button and select the "Activity Schedule Template Three Minute Timer.png" or drag the .png file into specified area.

16. Press "Done." This now makes a template for a timer page that cannot be edited or moved.

17. Click "Insert image" icon (Fig. 8), resize, and place a picture of an open-ended/timed activity on the page above the circles (as shown in Fig. 19).

\section{Creating Navigation for a Three Minute Timer Page}

1. Draw a large rectangle using the "Shape" tool (Fig. 9) over the entire page.

2. Click on the large rectangle and navigate to the "Insert Link" icon (Fig. 10) in the toolbar. A white pop-up bar will appear on the screen. Click on "Slides in this presentation" and select the current slide number. Press "Apply."

3. Click on the large rectangle and change "Color fill" and "Border color" (Fig. 11) to transparent.

4. Create smaller dots (circles) on the corresponding timer buttons (Fig. 19).

a. Select "Shape" tool (Fig. 9) and draw one small button-sized red circle. (Pressing shift while drawing the circle will keep it perfectly round.) Right click on the circle then select "Copy" and "Paste" once. Use "Color fill" icon (Fig. 3) to change the second dot to green.

i. Move the red dot on top of the timer's minute button.

ii. Move the green dot on top of the timer's start button.

5. Right click the timer page in left navigation bar. Select "Copy."

6. "Paste" four more timer pages into the left navigation bar (similar to Fig. 17).

7. Create a new blank page by clicking on the previous page (the final timer page) in the left navigation bar and pressing the "Enter" key.

8. Navigate to the first timer slide.

9. Right click on green button dot. Navigate to "Order," then "Send to Back." 
10. Select the red button dot. Navigate to "Insert Link" icon (Fig. 10) in the toolbar. Click on "Slides in this presentation" and select "Next Slide." Press "Apply."

11. Repeat steps 9-10 for the next two timer slides.

12. On the fourth timer slide right click on the red dot. Navigate to "Order" then "Send to Back."

13. Select the green button dot. Navigate to "Insert Link" icon (Fig. 10) in the toolbar. Click on "Slides in this presentation" and select "Next Slide." Press "Apply."

14. Navigate back to the second timer slide. Select "Text box" icon (Fig. 14), create a text box, and type 1:00. Place the time text box over the timer screen. Adjust text box size, font size, and text box color fill as needed.

15. Select text box with 1:00. "Copy" the text box and "Paste" it to the remaining timer pages. Edit the text boxes on each subsequent timer page to increase the time shown by one minute (Fig. 23). Make the timer text boxes on the fourth and fifth timer pages to both say 3:00.
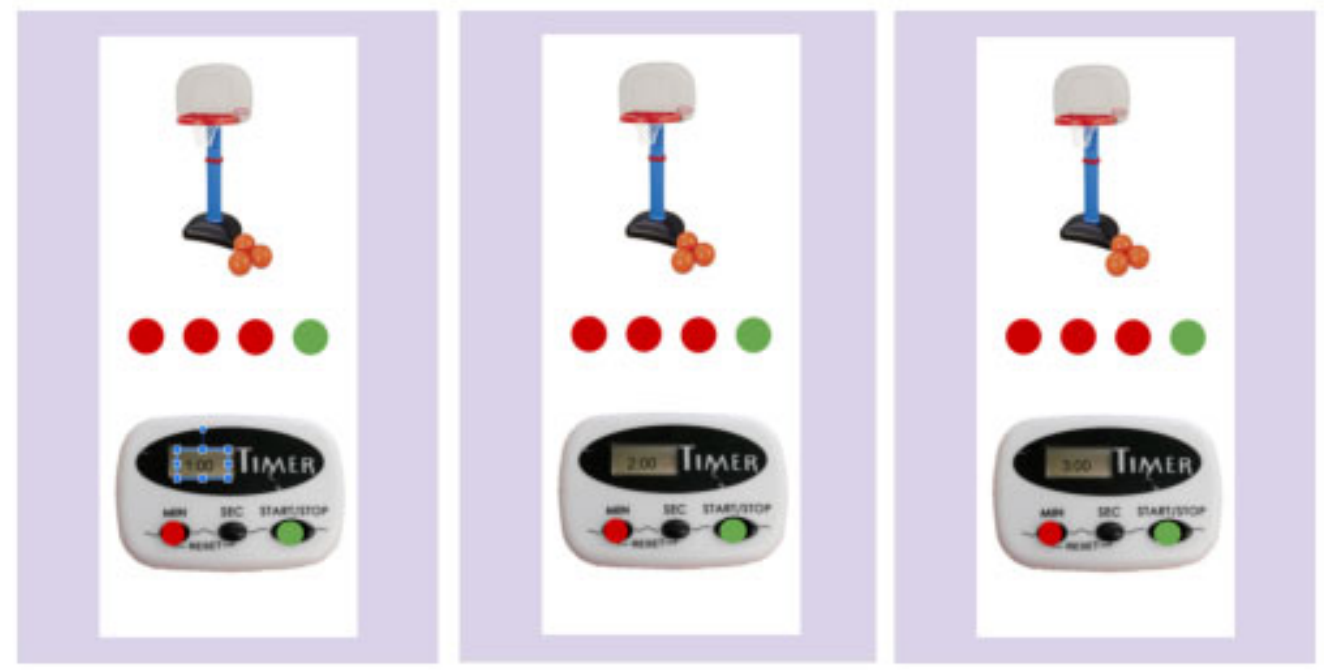

Fig. 23 Timers with time on screen

16. After copying and editing the text boxes, right click the timer text box on each page and click "Order" then "Send to back."

17. Select the fifth timer slide in the left navigation bar.

18. Click on "Insert" menu (Fig. 24) and select "Video."

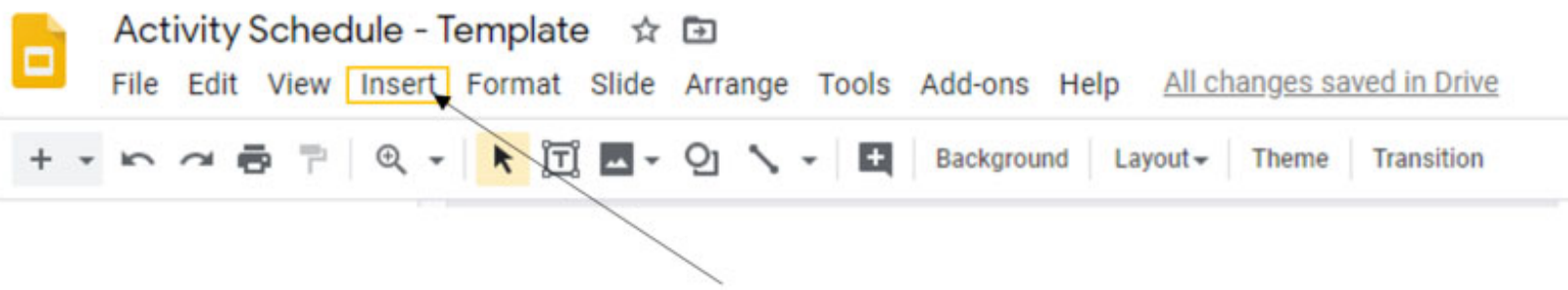

Fig. 24 Insert icon

19. A YouTube search bar will appear. Type in "3 minute timer." Choose timer and press "Select" button. 
20. A "Format options" bar will be present on the right when the video is inserted. Select "Autoplay when presenting" as seen in Fig. 25.
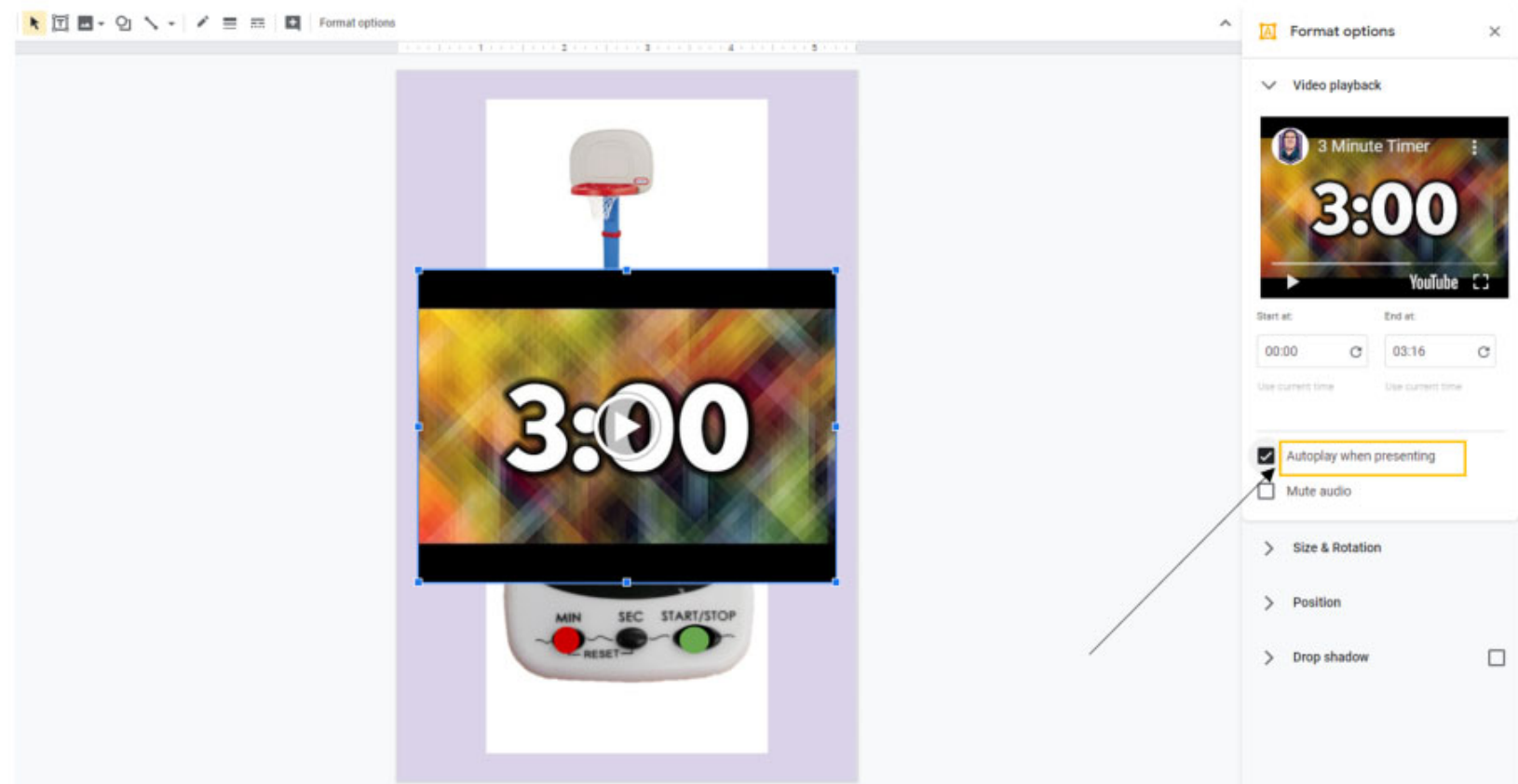

[] Format options

Fig. 25 Video Format options with autoplay when presenting option

21. Select "Next arrow" from the cover page of the presentation (with Student's Name).

"Copy" and "Paste" the arrow in the bottom right of the video page (Fig. 26).

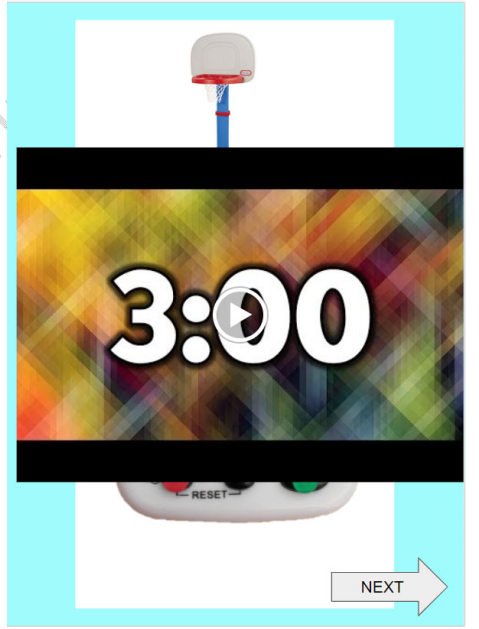

Fig. 26 Next arrow on video page

22. After the timer video page, create a blank slide to continue making the schedule.

a. To finish the schedule, see Creating a New Schedule/Modifying the Schedule Template.

23. Go to "Present" icon (Fig. 12), located on top right corner of tool bar, to view and navigate timed activity slides. 
a. Navigate through the presentation by clicking on the timer dots in sequence and the "Next arrow." Each click should advance to the next slide in the sequence.

i. If the slides do not advance correctly, check the links by clicking on the linked object. When clicked on, each linked object should display a white link bar below with the words Next slide. If the slide linked to the object is incorrect (or missing), click "Remove link" and return to the step corresponding to the specific object:

1. Red dot - Step 10 in Creating Navigation for a Three Minute Timer Page

2. Green dot - Step 13 in Creating Navigation for a Three Minute Timer Page

3. Next arrow - Step 21 in Creating Navigation for a Three Minute Timer Page

b. After all transitions are working correctly in sequence, restart the slideshow. Check that no other transitions occur when clicking elsewhere on the screen (anywhere other than the linked objects).

i. If transitions occur, check the following:

1. Ensure that the transparent rectangle is linked correctly. If it is not, return to Step 2 in Creating Navigation for a Three Minute Timer Page.

2. If an incorrect object is creating a transition, exit slideshow and navigate to the slide with that object. Right click on the object, select "Order," and "Send to back."

\section{Creating a New Schedule/Modifying the Schedule Template}

To create a new schedule, "Copy" and "Paste" the template slide sets into a new Google Slides presentation. The template sequences will maintain links and other properties. For example, to create one choice activity, copy all five slides of the choice page. This task analysis will assist in creating a template that clinicians can then use to create client specific schedules. Each schedule should end with a terminal reinforcer that is linked to a blank page, signaling the end of the schedule (see Appendix 3). To replace pictures in any of the pages, select the large transparent rectangle and move it out of the way. Then edit/replace pictures and place the large 
transparent rectangle back in place. To test and ensure all pages and features are working correctly, we suggest selecting the "Present" button (Fig. 12) to navigate and interact with the slides as the client would to check for errors. To assist with following the activity schedule template sequence, we provide a flow chart example in Appendix 3.

\section{Sharing Schedules with Families and Clients}

There are various options for sharing activity schedule files with caregivers and clients. One way is to send the caregivers a link to the activity schedule file. To create a link, select the yellow "Share" button (Fig. 27) in the top right corner. A pop-up menu will appear that provides sharing options, including view-only or editable access. Select "Get shareable link" and then email this link to the family/client. Other examples of how to share the activity schedule include creating a Google Drive folder with multiple schedule variations or sharing with other instructional materials using Google Classroom. For all sharing options, learners will need to view the activity schedules in "Present" form or slideshow view. To access on mobile devices or tablets, they will need to download the Google Slides app.

Activity Schedule - Template is $⿴$

File Edit View Insert Format Slide Arrange Tools Add-ons Help Allchanges saved in Drive

므 Present

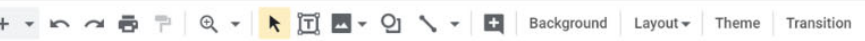

(1)

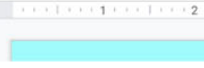

Fig. 27 Share button

\section{Discussion}

Researchers have demonstrated that photographic activity schedules can be an effective tool to promote independence and reduce the need for adult prompts across a variety of behaviors and for many different types of learners. Whereas much of this body of research has investigated the use of binder-based or single-page paper activity schedules, in recent years, 
researchers have demonstrated that technology-based digital schedules can also be effective and, in some circumstances, preferred by learners (Giles \& Markham, 2017). Digital activity schedules have multiple potential advantages that make them well-suited to situations such as the current COVID-19 crisis, during which face-to-face contact between behavior analysts and their clients and families may be reduced or eliminated. Another possible advantage includes the relative ease with which they can be modified and the potential for behavior analysts to create and disseminate digital activity schedules electronically. The task analysis included in this paper may be a useful tool as behavior analysts create and disseminate digital activity schedules. 


\section{References}

Akers, J. S., Higbee, T. S., Gerencser, K. R., \& Pellegrino, A. J. (2018). An evaluation of group activity schedules to promote social play in children with autism. Journal of Applied Behavior Analysis, 51, 553-570. https://doi.org/10.1002/jaba.474

Betz, A., Higbee, T. S., \& Reagon, K. A. (2008). Using joint activity schedules to promote peer engagement in preschoolers with autism. Journal of Applied Behavior Analysis, 41, 237241. https://doi.org/10.1901/jaba.2008.41-237

Brodhead, M. T., Higbee, T. S., Pollard, J. S., Akers, J. S., \& Gerencser, K. R. (2014). The use of linked activity schedules to teach children with autism to play hide-and-seek. Journal of Applied Behavior Analysis, 47, 645-650. https://doi.org/10.1002/jaba.145

Carlile, K. A., Reeve, S. A., Reeve, K. F., \& DeBar, R. M. (2013). Using activity schedules on the iPod touch to teach leisure skills to children with autism. Education and Treatment of Children, 36(2), 33-57. https://doi.org/10.1353/etc.2013.0015

Carson, K. D., Gast, D. L., \& Ayres, K. M. (2008). Effects of a photo activity schedule book on independent task changes by students with intellectual disabilities in community job sites. European Journal of Special Needs Education, 23, 269-279. https://doi.org/10.1080/08856250802130475

Dauphin, M., Kinney, E. M., \& Stromer, R. (2004). Using vide0-enhanced activity schedules and matrix training to teach sociodramatic play to a child with autism. Journal of Positive Behavior, 6, 238-250. https://doi.org/10.1177/10983007040060040501 
Gerencser, K.R., Higbee, T.S., Akers, J.S., \& Contreras, B.P. (2017). Evaluation of interactive computerized training to implement photographic activity schedules with children with autism spectrum disorder. Journal of Applied Behavior Analysis, 50, 567-581. https://doi.org/ 10.1002/jaba.386

Giles, A. \& Markham, V. (2017). Comparing book- and tablet-based picture activity schedules: Acquisition and preference. Behavior Modification, 41, 647-664. https://doi.org/ $10.1177 / 0145445517700817$

Gourwitz, J. R. (2014). IPads for students with ASD: Comparing delivery modes for visual activity schedule (Doctoral dissertation). http://etd.fcla.edu/ CF/CFE0005339/Gourwitz_Jillian_R_201408_PhD.pdf

Higbee, T.S. \& Brodhead, M.T. (2016). Promoting independence, verbal behavior, and social skills in individuals with autism through activity schedules and script fading. International Journal of Behavior Analysis and Autism Spectrum Disorders, 1, 1-8.

Higbee, T.S. \& Sellers, T.P. (2017). Visual learning strategies to promote independence and appropriate social behavior. In J.B. Leaf (Ed.), Handbook of Social Skills and Autism Spectrum Disorder: Assessment, Curricula, and Interventions. Springer.

MacDuff, G. S., Krantz, P. J., \& McClannahan, L. E. (1993). Teaching children with autism to use photographic activity schedules: Maintenance and generalization of complex response chains. Journal of Applied Behavior Analysis, 26, 89-97. https://doi.org/10.1901/jaba.1993.26-89

McClannahan, L. E., \& Krantz, P. J. (1999). Activity schedules for children with autism: Teaching independent behavior. Bethesda, MD: Woodbine House. 
Rehfeldt, R. A., Kinney, E. M., Root, S., \& Stromer, R. (2004). Creating activity schedules using

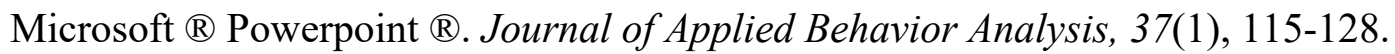
https://doi.org/10.1901/jaba.2004.37-115 


\section{Appendix 1: Example of an activity schedule data sheet}

Activity Schedule Following Data Sheet

Learner :

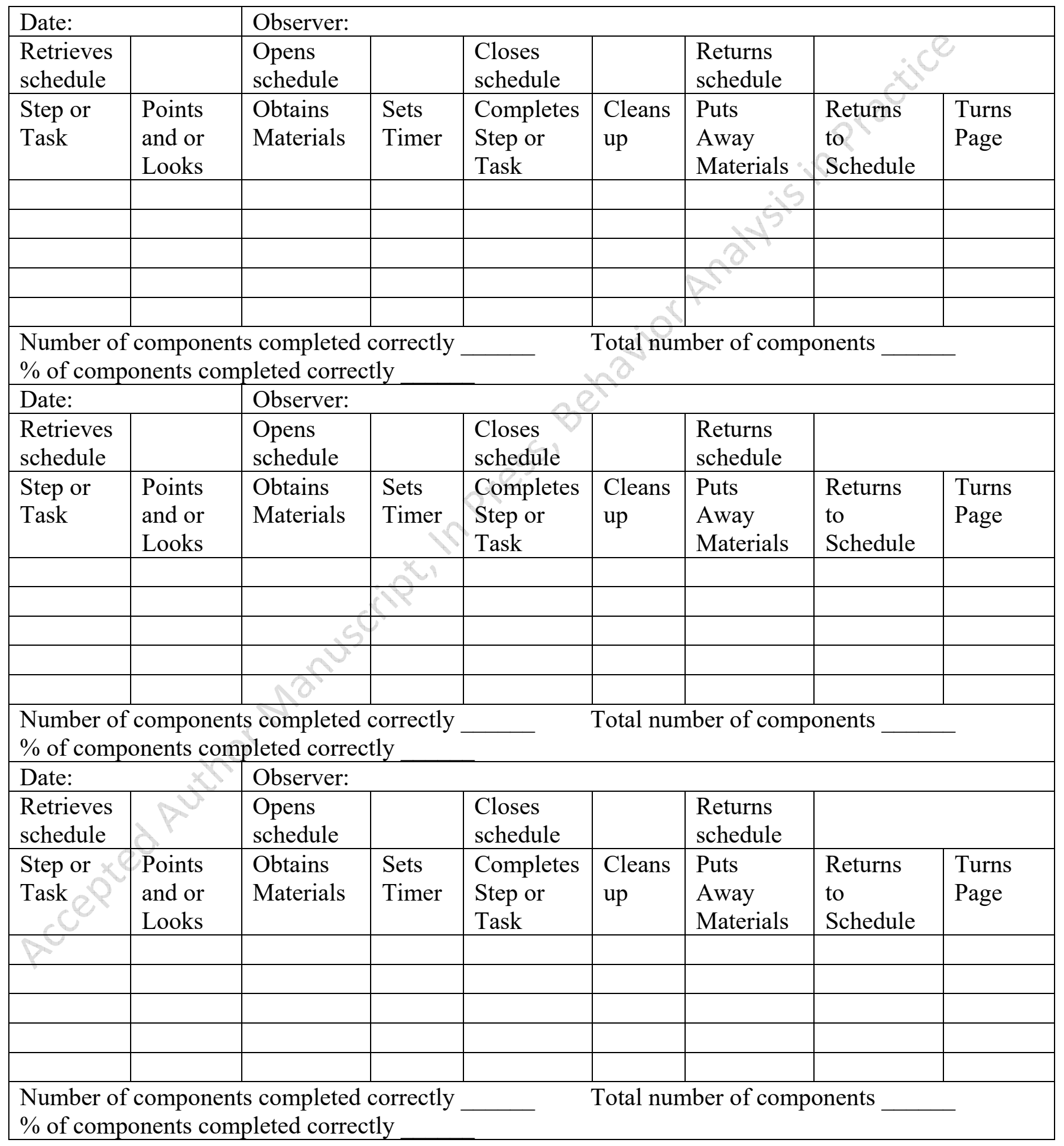




\section{Appendix 2: Data sheet similar to that used in the Gerencser et al., 2017 Study}

\section{Activity Schedule: Test Session}

Parent:

Learner:

Date:

Directions: Collect data on the learner's performance. Correct responses will be scored as a plus (+) and incorrect response or responses you provided physical supports will be scored as a minus ( $)$.

\begin{tabular}{|c|c|c|c|c|c|}
\hline $\begin{array}{c}\text { Activity Schedule } \\
\text { Steps }\end{array}$ & \multicolumn{5}{|c|}{ Performance $(+$ or -$)$} \\
\hline \multicolumn{6}{|l|}{ Retrieves schedule } \\
\hline \multicolumn{6}{|l|}{ Opens schedule } \\
\hline & Page 1: & Page 2: & Page 3: & Page 4: & Page 5: \\
\hline \multicolumn{6}{|l|}{ Points to picture } \\
\hline \multicolumn{6}{|l|}{ Obtains material } \\
\hline \multicolumn{6}{|l|}{$\begin{array}{l}\text { Completes } \\
\text { activity/consumes treat }\end{array}$} \\
\hline \multicolumn{6}{|l|}{$\begin{array}{l}\text { Cleans up/return } \\
\text { material }\end{array}$} \\
\hline \multicolumn{6}{|l|}{ Returns to schedule } \\
\hline \multicolumn{6}{|l|}{ Turns page } \\
\hline \multicolumn{6}{|l|}{ Closes schedule } \\
\hline \multicolumn{6}{|l|}{ Returns schedule } \\
\hline$+=$ Independent & & \# of indep & $\mathrm{nt} /$ tota & 1 & $=\ldots$ \\
\hline
\end{tabular}


Appendix 3: Activity Schedule Template Flow Chart

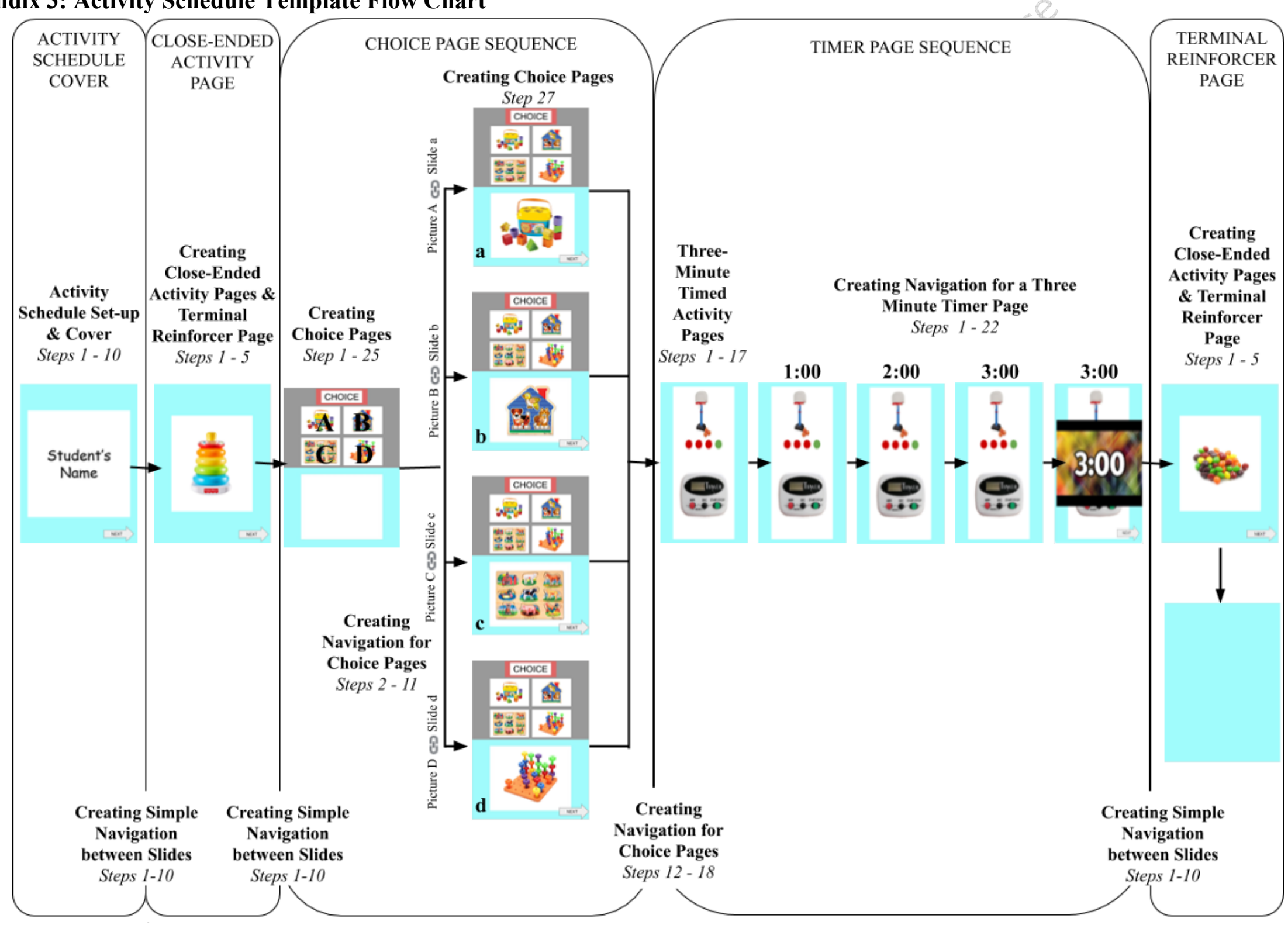


Appendix 4: Sample timer images to use
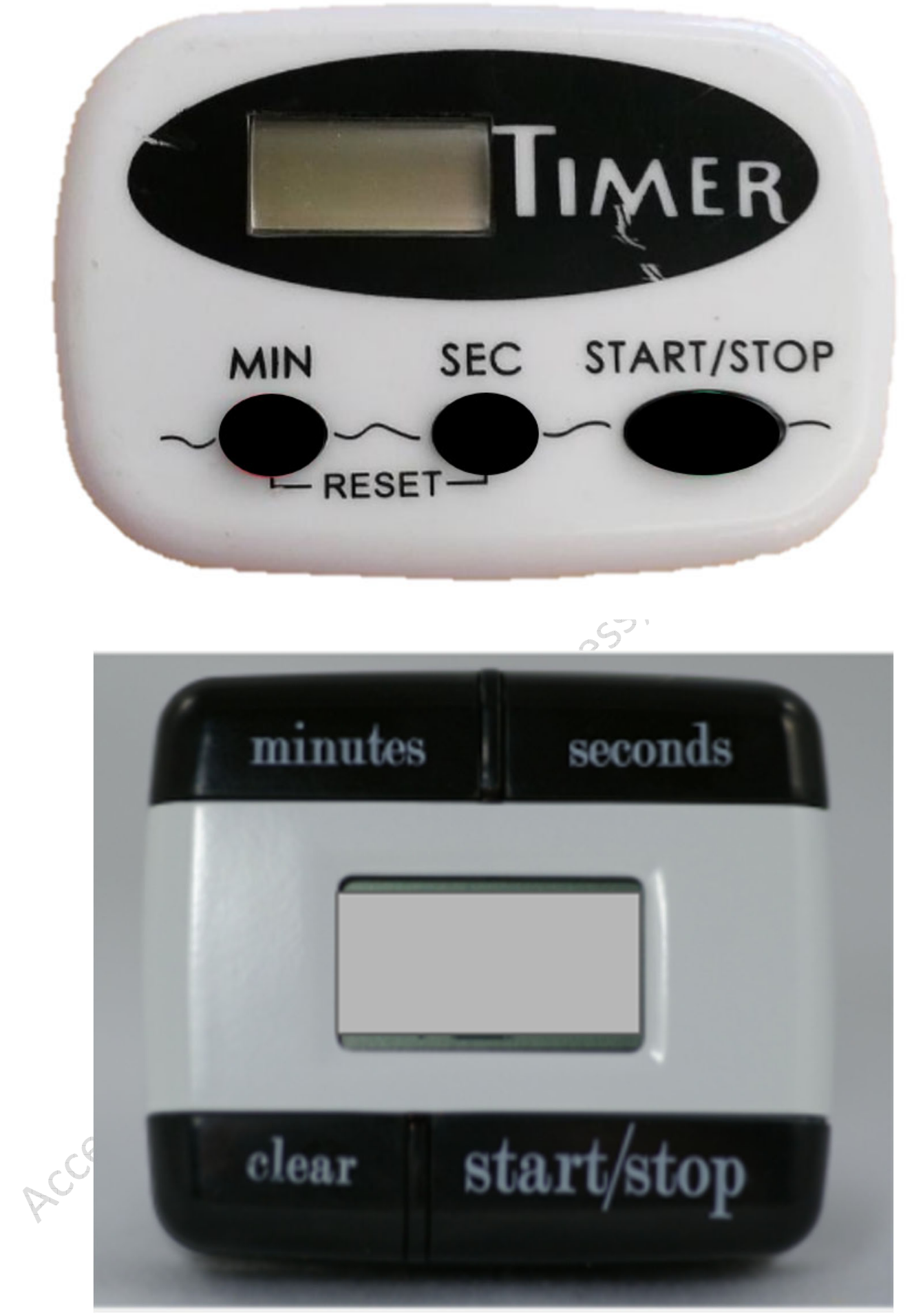